\title{
ON THE PRIME NUMBER LEMMA OF SELBERG
}

\author{
FILIP SAIDAK
}

Dedicated to Atle Selberg - at the beginning of his 10th decade.

\begin{abstract}
The key result needed in almost all elementary proofs of the Prime Number Theorem is a prime number lemma proved by Atle Selberg in 1948. Without restricting ourselves to purely elementary techniques we show how the error term in Selberg's fundamental lemma relates to the error term in the Prime Number Theorem. In spite of all the interest in this topic over the last sixty years this particular question seems to have been overlooked in the past.
\end{abstract}

\section{PRIME NUMBER THEOREM}

Let

$$
\pi(x):=\sum_{p \leq x} 1 .
$$

In its simplest form the Prime Number Theorem (PNT) asserts that, as $x \rightarrow \infty$,

$$
\pi(x) \sim \lim _{\epsilon \rightarrow 0}\left\{\int_{0}^{1-\epsilon}+\int_{1+\epsilon}^{x}\right\} \frac{d t}{\log t} \Longleftrightarrow \psi(x) \sim x,
$$

where $\psi(x)=\sum_{n \leq x} \Lambda(n)=\sum_{p^{m} \leq x} \log p$. The important error function $E(x)$,

$$
E(x):=\psi(x)-x
$$

is closely connected to zero-free regions of $\zeta(s)$ in the critical strip. In fact, since

$$
\frac{\psi(x+0)+\psi(x-0)}{2}-x=-\sum_{\rho} \frac{x^{\rho}}{\rho}-\frac{\zeta^{\prime}}{\zeta}(0)-\frac{1}{2} \log \left(1-\frac{1}{x^{2}}\right)
$$

and $\sum_{0<\Im(\rho)<T} \frac{1}{|\rho|}=O(\log T)^{2}$, both sums being extended over all non-trivial zeros of $\zeta(s)$, it is easy to see that (e.g. [5], §18): $E(x)=O\left(x^{\Delta}(\log x)^{2}\right)$ if $\zeta(s) \neq 0$ for $\Re(s)>\Delta$. However as Selberg [19] and Erdős [7] discovered in 1948, analytic methods are not exclusively needed for estimation of $E(x)$ or the proof of PNT.

\section{SELBERG'S LEMMA}

The following remarkable lemma of Atle Selberg is used in almost all known elementary proofs of (1):

Lemma 2.1 (Selberg [19], p. 309)). For all $x>0$ we have

$$
\psi(x) \log x+\int_{1}^{x} \psi\left(\frac{x}{t}\right) d \psi(t)=2 x \log x+O(x) .
$$

1991 Mathematics Subject Classification. 11N05, 11N37, 11M06.

Key words and phrases. prime numbers, the prime number theorem, Selberg's formula. 
In his original proof of Lemma 2.1 (see [19], p. 307) Selberg used only the rough elementary Chebyshev-type bound $\vartheta(x):=\sum_{p \leq x} \log p=O(x)$, which is equivalent to the weak estimates $\pi(x)=O(x / \log x)$ and $\psi(x)=O(x)$. It seems natural to ask whether a somewhat better estimate for $\psi(x)$ could give us a sharper result than (2). Moreover, if we are willing to abandon the idea of necessity of purely elementary means, and just look at Selberg's lemma as a fundamental result concerning prime numbers worth studying in its own right, then it seems of interest to investigate the question of what the sharpest possible estimates concerning $\psi(x)$ may yield in this direction. This "converse" problem is exactly what we investigate below.

The purpose of this paper is to give a simple proof the following:

THEOREM 2.2. If we assume that $\psi(x)=x+E(x)$, then we have

$$
\psi(x) \log x+\int_{1}^{x} \psi\left(\frac{x}{t}\right) d \psi(t)=2 x \log x-(2 \gamma+1) x+O\left(E(x)(\log x)^{2}\right) .
$$

Corollary 2.3. The sharpest known version of the Prime Number Theorem (still due to Korobov [8] and Vinogradov [22], from 1958) implies that there exists a constant $A>0$, such that for all $\epsilon>0$ we have

$$
\begin{aligned}
\psi(x) \log x+\int_{1}^{x} \psi\left(\frac{x}{t}\right) d \psi(t) & =2 x \log x-(2 \gamma+1) x \\
& +O\left(x \exp \left(-\frac{A(\log x)^{3 / 5}}{(\log \log x)^{1 / 5}}\right)\right) .
\end{aligned}
$$

COROLlary 2.4. Under the assumption of the Riemann Hypothesis [17]

$$
\psi(x) \log x+\int_{1}^{x} \psi\left(\frac{x}{t}\right) d \psi(t)=2 x \log x-(2 \gamma+1) x+O\left(\sqrt{x}(\log x)^{3}\right) .
$$

Remark 2.5. Starting with Kuhn [9] and van der Corput [4] many results concerning the error (remainder) term in the Prime Number Theorem by elementary methods have been published over the last six decades, including the works of Bombieri [2], Wirsing [23], Diamond \& Steinig [6], Lavrik \& Sobirov [10] and Lu [13], to mention just a few. Moreover, various extensions and generalizations of Lemma 2.1 were obtained by Shapiro [20], Tatuzawa \& Iseki [21], Popken [15], Breusch [3] and others. However these contributions did not involve sharpening of Selberg's lemma per se, and they did not explicitly study the relationship of its error term to that of the error in the Prime Number Theorem either.

REMARK 2.6. The sharpest explicit version of (2) is due to V. Nevanlinna [14]:

$$
\psi(x) \log x+\int_{1}^{x} \psi\left(\frac{x}{t}\right) d \psi(t)=2 x \log x-(2 \gamma+1) x+o(x) .
$$

\section{LEMMAS}

The proof of our main theorem is short and straight-forward, but it relies on some basic lemmas which, for convenience, we list below with appropriate explanations.

For all $x \in \mathbb{R}$, we denote by $[x]$ the integer part of $x$. 
Lemma 3.1 (Euler). Let $\gamma:=\sum_{n=2}^{\infty}(-1)^{n} \frac{\zeta(n)}{n}=0.5772156649 \cdots=-\Gamma^{\prime}(1)$ be Euler's constant. Then, for all $x>0$, we have

$$
\sum_{n \leq x} \frac{1}{n}=\log x+\gamma+O\left(\frac{1}{x}\right) \text { and } \sum_{n \leq x} \log n=x \log x-x+O(\log x) .
$$

Proof. Both results follow immediately from simple applications of the EulerMacLaurin Summation, see [1] - Theorem 2.4 and Theorem 2.3, respectively.

Lemma 3.2. For positive integers $n$, define the Möbius function $\mu(n)$ as

$$
\mu(n):= \begin{cases}1 & \text { if } \mathrm{n}=1 \\ (-1)^{k} & \text { if } \mathrm{n}=\mathrm{p}_{1} \mathrm{p}_{2} \cdots \mathrm{p}_{\mathrm{k}} \\ 0 & \text { if } \mathrm{p}^{2} \mid \mathrm{n}\end{cases}
$$

Then

$$
\psi(x)-x \ll E(x) \Longrightarrow M(x):=\sum_{n \leq x} \mu(n) \ll E(x) .
$$

Proof. In fact, under the given assumption, even a little bit stronger bound on $M(x)$ could be proved - see Landau [12], Vol. II, Chapters 10-12.

For the Chebyshev function $\psi(x)$ we will need the following two lemmas:

Lemma 3.3. Let $\psi(x)=x+E(x)$, then for $x>1$ we have

$$
\int_{1}^{x} \frac{d \psi(t)}{t}=\log x-\gamma+O\left(\frac{E(x)}{x}\right) .
$$

Proof. For all real $x>1$, integrating by parts gives us

$$
\begin{aligned}
\int_{1}^{x} \frac{d \psi(t)}{t} & =\frac{\psi(x)}{x}-\int_{1}^{x} \frac{-\psi(t)}{t^{2}} d[t]=\frac{x+E(x)}{x}+\int_{1}^{x} \frac{t+E(t)}{t^{2}} d[t] \\
& =1+\int_{1}^{x} \frac{1}{t} d[t]+O\left(\frac{E(x)}{x}\right)=\log x-\gamma+O\left(\frac{E(x)}{x}\right),
\end{aligned}
$$

by Lemma 3.1.

Lemma 3.4 (Tatuzawa \& Iseki [21]). Consider two real valued functions $f(x)$ and $g(x)$ related via the identity

$$
g(x)=\log x \sum_{n \leq x} f\left(\frac{x}{n}\right) .
$$

Then, for all $x>1$, we have

$$
f(x) \log x+\int_{1}^{x} f\left(\frac{x}{n}\right) d \psi(t)=\int_{1}^{x} g\left(\frac{x}{t}\right) d M(t) .
$$

Corollary 3.5 (Nevanlinna [14]). The function $\psi(x)$ can be written in the form

$$
\psi(x)=\int_{1}^{x}\left[\frac{x}{t}\right] \log \frac{x}{t} d M(t)-\log x .
$$

Proof. Take $f(x)=1$ in (5). Then $g(x)=[x] \log x$ and the result is clear. 


\section{PROOF OF THE THEOREM}

The key idea is to compare the two sides of Lemma 3.4 when $f(x)=\psi(x)-x$. With this particular choice of $f(x)$, the left hand side (LHS) of (5) becomes

$$
\begin{aligned}
\text { LHS } & :=(\psi(x)-x) \log x+\int_{1}^{x}\left(\psi\left(\frac{x}{t}\right)-\frac{x}{t}\right) d \psi(t) \\
& =\psi(x) \log x+\int_{1}^{x} \psi\left(\frac{x}{t}\right) d \psi(t)-x \log x-x \int_{1}^{x} \frac{d \psi(t)}{t} \\
& =\psi(x) \log x+\int_{1}^{x} \psi\left(\frac{x}{t}\right) d \psi(t)-2 x \log x+\gamma x+O(E(x)),
\end{aligned}
$$

by Lemma 3.3. On the other hand, the two estimates in our Lemma 3.1 imply

$$
\begin{aligned}
g(x) & =\log x \sum_{n \leq x}\left(\psi\left(\frac{x}{n}\right)-\frac{x}{n}\right)=\log x \sum_{n \leq x} \log n-x \log x \sum_{n \leq x} \frac{1}{n} \\
& =\left(x \log ^{2} x-x \log x+O(\log x)^{2}\right)-\left(x \log ^{2} x+\gamma x \log x+O(\log x)\right) \\
& =-(\gamma+1)[x] \log x+h(x) \log x,
\end{aligned}
$$

where $h(x)$ is a function that satisfies the bound $h(x)=O(\log x)$. Therefore, the right hand side (RHS) of (5) can be now rewritten as:

$$
\begin{aligned}
\mathrm{RHS} & :=\int_{1}^{x} g\left(\frac{x}{t}\right) d M(t) \\
& =-(\gamma+1) \int_{1}^{x}\left[\frac{x}{t}\right] \log \frac{x}{t} d M(t)+\int_{1}^{x} h\left(\frac{x}{t}\right) \log \frac{x}{t} d M(t) \\
& =-(\gamma+1)(\log x+\psi(x))+O\left((\log x)^{2} \int_{1}^{x} d M(t)\right) \\
& =-(\gamma+1) x+O(E(x))+O\left(E(x)(\log x)^{2}\right),
\end{aligned}
$$

by Lemma 3.2 and Corollary 3.5. Equating the two sides proves the theorem.

\section{ACKNOWLEDGEMENTS}

I would like to thank the referee for several helpful comments.

\section{REFERENCES}

1. Ayoub, R, - An Introduction to the Analytic Theory of Numbers, Chapter 2, AMS Math. Surveys, No. 10, 1963.

2. Bombieri, E. - Maggiorazione del resto nel "Primzahlsatz" col metodo di Erdös-Selberg, Ist. Lombardo Accad. Sci. Lett. Rend. A, 96, 343-350, 1962.

3. Breusch, R. - Elementary proof of the prime number theorem with remainder term, Pacific J. Math., 10 (1960), 487-497.

4. van der Corput, G. J. - Sur le reste dans la démonstration élémentaire du théorème des nombres premiers, Colloque sur la Théorie des Nombres, Bruxelles, 1955, 163-182.

5. Davenport, H. - Multiplicative number theory, 3rd Ed., GTM 74, Springer, New York, 2000.

6. Diamond, H. G. \& Steinig, J. - An elementary proof of the prime number theorem with a remainder term, Invent. Math., 11, 1970, 199-258.

7. Erdős, P. - On a new method in elementary number theory which leads to an elementary proof of the prime number theorem, Proc. Nat. Acad. Sci. U. S. A., 35, 374-384, 1949.

8. Korobov, N. M. - Estimates for trigonometric sums and their applications, Uspehi Mat. Nauk, 13 (1958), 185-192. 
9. Kuhn, P. - Eine Verbesserung des Restgliedes beim elementaren Beweis des Primzahlsatzes, Math. Scand., 3 (1955), 75-89.

10. Lavrik, A. F. \& Sobirov, A. S. - On the remainder term in the elementary proof of the prime number theorem, Doklady Akad. Nauk SSSR, 211 (1973), 534-536.

11. Landau, E. - Handbuch der Lehre von der Verteilung der Primzahlen, Teubner, Leipzig, 1909. (Reprinted 1974 by Chelsea, New York.)

12. Landau, E. - Vorlesungen über Zahlentheorie, Vol. II, Chelsea, New York, 1947.

13. Lu, W. C. - On the elementary proof of the prime number theorem with a remainder term, Rocky Mountain J. Math., 29 (1999), no. 3, 979-1053.

14. Nevanlinna, V. - A refinement of Selberg's asymptotic equation, Pacific J. Math., Vol. 21, No. 3, 1967.

15. Popken, J. - On convolutions in number theory, Indag. Math., 17, (1955), 10-15.

16. Postnikov, A. G. \& Romanov, N. P. - A simplification of A. Selberg's elementary proof of the asymptotic law of distribution of prime numbers, Uspehi Mat. Nauk (N.S.) 10 (1955), no. 4, (66), 75-87.

17. Riemann, B. - Über die Anzahl der Primzahlen unter einer gegebenen Grösse (1859), Collected Works, p. 145, Teubner, Leipzig, 1892.

18. Saidak, F. - An elementary proof of a theorem of Delange, C. R. Math. Acad. Sci. Soc. R. Can., Vol. 24, 4, 144-151, 2002.

19. Selberg, A. - An elementary proof of the prime-number theorem, Ann. of Math. (2), 50, 305-313, 1949.

20. Shapiro, H. - On a theorem of Selberg and generalizations, Ann. of Math. (2) 51, (1950), 485-497.

21. Tatuzawa, T. \& Iseki, K. - On Selberg's elementary proof of the prime-number theorem, Proc. Japan Acad., 27, 340-342, 1951.

22. Vinogradov, I. M. - A new estimate for $\zeta(1+i t)$, Izvestia Akad. Nauk SSSR, Ser. Mat. 22 (1958), 161-164.

23. Wirsing, E. - Elementare Beweise des Primzahlsatzes mit Restglied. I. \& II., J. Reine Angew. Math., 211, 205-214, 1962, and 214/215, 1-18, 1964.

Department of Mathematics, University of North Carolina, Greensboro, NC 27401, U.S.A.

E-mail: f_saidak@uncg.edu 